\title{
FULL RANGE BOUNDEDNESS OF BILINEAR HILBERT TRANSFORM ALONG CERTAIN POLYNOMIALS
}

\author{
DONG DONG
}

Abstract. Let $P$ and $Q$ be two polynomials without constant term. Assume that the operator $B_{P, Q}(f, g)(x)=\int f(x-P(t)) g(x-Q(t)) \frac{d t}{t}$ is bounded from $L^{p_{1}} \times L^{p_{2}}$ into $L^{r}, p_{1}, p_{2} \in$ $(1, \infty), \frac{1}{p_{1}}+\frac{1}{p_{2}}=\frac{1}{r}$. It is proved that if $P^{\prime}(t)>0$ for all $t \neq 0$, then $r \geqslant \frac{d}{d+1}$. Here $d$ is the correlation degree of $P$ and $Q$ which is defined as the largest multiplicity of non-zero real roots of $P^{\prime}-Q^{\prime}$.

Mathematics subject classification (2010): 42B20, 47B38.

Keywords and phrases: Bilinear Hilbert transform, boundedness, correlation degree, full range.

\section{REFERENCES}

[1] D. Bilyk and L. Grafakos, Distributional estimates for the bilinear Hilbert transform, J. Geom. Anal. 16 (2006), no. 4, 563-584.

[2] D. Bily K AND L. GRAFAKos, A new way of looking at distributional estimates; applications for the bilinear Hilbert transform, Collect. Math. 2006, Vol. Extra, 141-169.

[3] M. Christ, A. Nagel, E. M. Stein And S. WAinger, Singular and maximal Radon transforms: analysis and geometry, Ann. of Math. (2) 150 (1999), no. 2, 489-577.

[4] F. Di Plinio And C. Thiele, Endpoint bounds for the bilinear Hilbert transform, Trans. Amer. Math. Soc. 368 (2016), no. 6, 3931-3972.

[5] D. Dong, On bilinear Hilbert transform along two polynomials, Proc. Amer. Math. Soc. (to appear), https://arxiv.org/abs/1708.01326.

[6] L. GRafakos AND X. LI, Uniform bounds for the bilinear Hilbert transforms, I, Ann. of Math. (2) 159 (2004), no. 3, 889-933.

[7] J. Guo And L. Xiao, Bilinear Hilbert transforms associated with plane curves, J. Geom. Anal. 26 (2016), no. 2, 967-995.

[8] M. Lacey and C. Thiele, $L^{p}$ estimates on the bilinear Hilbert transform for $2<p<\infty$, Ann. Math. 146 (1997), 693-724.

[9] M. LACEY AND C. THiele, On Calderón's conjecture, Ann. of Math. (2) 149 (1999), no. 2, 475-496.

[10] X. LI, Uniform bounds for the bilinear Hilbert transforms, II, Rev. Mat. Iberoam. 22 (2006), no. 3, 1069-1126.

[11] X. LI, Uniform estimates for some paraproducts, New York J. Math. 14 (2008), 145-192.

[12] X. LI, Bilinear Hilbert transforms along curves I: The monomial case, Anal. PDE 6 (2013), no. 1, $197-220$.

[13] X. Li AND L. XIAO, Uniform estimates for bilinear Hilbert transform and bilinear maximal functions associated to polynomials, Amer. J. Math. 138 (2016), no. 4, 907-962.

[14] V. LIE, On the boundedness of the bilinear Hilbert transform along "non-flat" smooth curves, Amer. J. Math. 137 (2015), no. 2, 313-363.

[15] V. LIE, On the Boundedness of The Bilinear Hilbert Transform along "non-flat" smooth curves. The Banach triangle case $\left(L^{r}, 1 \leqslant r<\infty\right)$, Rev. Mat. Iberoam. 34 (2018), no. 1, 331-353.

[16] E. M. Stein And S. Wainger, Problems in harmonic analysis related to curvature, Bull. Amer. Math. Soc. 84 (1978), no. 6, 1239-1295.

[17] C. Thiele, A uniform estimate, Ann. of Math. (2) 156 (2002), no. 2, 519-563. 